\title{
CC CRYOSTAT VAPOR PRESSURE
}

\section{THERMOMETERS}

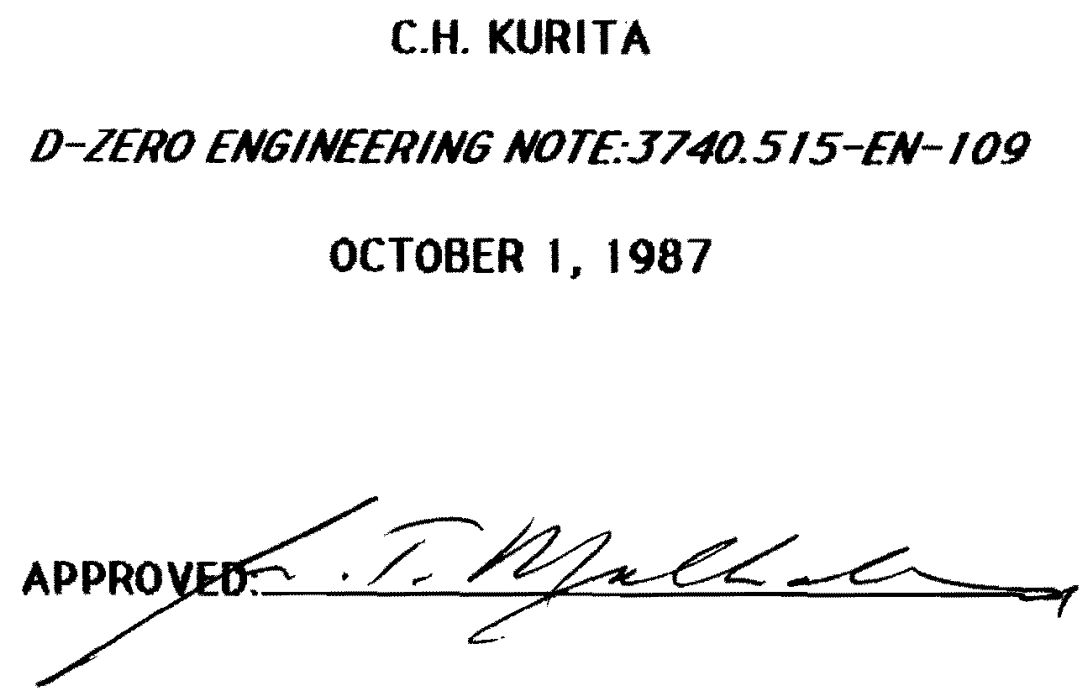




\section{INTRODUCTION}

vapor pressure thermometers will be used to measure the temperature of the liquid argon in the cryostat at two different levels. One bulb will be positioned near the top of the vessel, and a second bulb will be located near the bottom of the vessel. The volume of the bulbs is dependent upon the charge temperature and pressure chosen, the temperature range of the thermometer desired, the size and length of tubing used, and the warm volume involved

\section{BULB SIZING}

The charge temperature used in dimensioning the bulbs was $300 \mathrm{~K}$, and the charge pressure chosen was 10 atm (150 psig). A low charge pressure results in a small measurable temperature range. The lower the charge pressure, the more precise the pressure gauge reading, and therefore, the greater the accuracy of the temperature.

The size of tubing used has a 1/4 inch 0.D. with a ,049 inch wall thickness. Although this large tube diameter requires a greater bulb volume, it results in a faster vacuum pumping speed. The vacuum pumping speed is an important consideration if any water should get into the bulb and need to be pumped out. The lengths of the tubing used in the calculations for the top and bottom bulbs were 60 inches and 259 inches, respectively, dependent on the detailed positioning of the bulbs in the vessel. A 4-HW bushing (Parker Fluid Connectors) is used in the installation of the tubes.

The warm volume of the system is the volume of the pressure gauge, pressure transducer, and any outside tubing that does not experience a thermal gradient. The absolute pressure gauge used for calculations (Wallace and Tiernan series 1000) has a volume of $10 \mathrm{cc}$ in the pressure Bourdon system. The transducer used (Setra Systems model no. 204) has a pressure element volume of $0.590 \mathrm{cc}$.

The following formulas in the sizing of the bulb and are based on the assumption that the mass of the charge in the system remains constant. 


\title{
$M_{w v}=P_{b w} V_{b}+p_{t w} V_{t}+P_{g w} V_{g}$ \\ $M_{c v}=p_{b v} V_{b}+p_{t d} v_{t}+p_{g w} V_{g}$ \\ $M_{c l}=p_{b l} V_{b}+p_{t d} V_{t}+p_{g w} V_{g}$
}

(1)

(II)

(III)

\author{
$M=$ mass \\ $p=$ density \\ $v=$ volume \\ $w=$ warm \\ $v=$ vapor \\ $b=$ bulb \\ $t=$ tube \\ $g=$ gauge \\ $c=$ cold \\ $d=$ distributed \\ I=liquid \\ $v_{t}, v_{g}$ are constants
}

(1) $P_{b w}, p_{t w}, P_{g w}$ are constants given initial $P=10$ atm, $T=300 \mathrm{~K}$

(II) $p_{b v}, p_{t d}, p_{g w}$ are dependent on transition $P=P_{x}, T_{d}=200 K$

(III) $\mathrm{p}_{\mathrm{td}}, \mathrm{p}_{\mathrm{gw}}$ are constants given operating $\mathrm{P}=1 \mathrm{~atm}, T=87 \mathrm{~K}$

\section{$M_{w v}=M_{c v}=M_{c l}$}

Setting $(1)=(11)$ and solving for $V_{b}$

let $P_{x}=4 a t m$ (just above the intersection of vapor

pressure and ideal gas curves)

Solve for $p_{b 1}$ at 1 atm with $v_{b}$ value obtained

The fraction of liquid in the bulb, $x$, is found using:

$$
D_{D I}=(x)\left(p_{1}\right)+(1-x)\left(p_{g}\right)
$$


The conductance of the tube, $c_{t}$, is:

$$
\begin{aligned}
& C_{t}=182(D)^{4}\left(P_{\text {avg }}\right) / L_{t} \\
& D=\text { diameter }(\mathrm{cm}) \\
& P_{\text {avg }}=\text { average pressure in tube } \\
& L_{t}=\text { length of tube }(\mathrm{cm})
\end{aligned}
$$

The pumping speed, S, is:

$$
\begin{aligned}
& S=1 /\left(\left(1 / S_{0}\right)+\left(1 / C_{1}\right)+\left(1 / C_{2}\right)+\ldots\left(1 / C_{n}\right)\right) \\
& S_{0}=\text { pumping speed of pump } \gg>C_{t} \\
& C_{1}-C_{n} \text { are series conductances dominated by } C_{t} \\
& S \approx C_{t}
\end{aligned}
$$

The time constant for pumping out, $\hbar$ is:

$t=V / S$

allowing about 5 t to pump/purge argon

\section{SIZING RESULTS}

Using a tube length of 60 inches for the upper VPT and 259 inches for the lower VPT, the following values were obtained:

Upper VPT:

$$
\begin{aligned}
& v_{b}=3.004037 \mathrm{in}^{3} \\
& 5 t=244 \mathrm{~min} \\
& x=1.35 \%
\end{aligned}
$$


Lower VPT:

$$
\begin{aligned}
& v_{b}=8.248536 \mathrm{in}^{3} \\
& 5 t=3,022.71 \mathrm{~min} \\
& x=1.41 \%
\end{aligned}
$$

\section{BULB DESIGN}

Each bulb is to be fabricated from 304 stainless steel one inch diameter schedule 10 pipe and two one inch diameter schedule 10 pipe caps. Details can be found in DWG. 3740.210-MA-2224761 and DWG. $3740.210-M B-2223762$.

\section{CONCLUSION}

Using the principle that the mass within each VPT system remains constant, design detalls for the bulbs were established. Further calculations showed that the required pump/purge time does not pose a problem, nor does the liquid fraction of argon in the bulb. 


\title{
CC CRYOSTAT VAPOR PRESSURE THERMOMETERS
}

\author{
J.B. FITZPATRICK
}

D-ZERO ENGINEERING NOTE:3740.515-EN-109-A

APPENDIX A

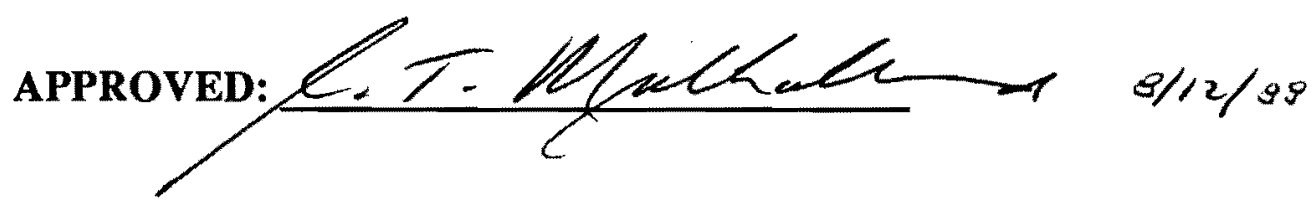


INTRODUCTION:

The purpose of this engineering note appendix is to document all changes that have been made to the design, and location of the $\mathrm{CC}$ Cryostat vapor pressure thermometers (VPT's).

\section{LOCATION / DESIGN:}

The final locations for VPT's are shown on D-Zero drawing \#3740.214.-MD-273239. Mounting configurations are referenced as well.

VPT \#1 - is located on the centerline of the bottom of the vessel. (port is denoted VPT1) The design has been changed from a bulb design to a 1" schedule 40 pipe cap design (see ref \#1) that is welded directly to the shell of the vessel. The bulb design was reconsidered due to concern that liquid might condense in the $1 / 4$ " line that resides in the vacuum space, under the superinsulation ( ie.. no insulation between inner wall and VPT tube) (ref \#2) The geometry of the tube requires that it make a sharp bend as it exits port VPT1 and heads up the vacuum space towards the top of the vessel. At this sharp bend, there is concern that enough liquid may condense that the reading is not that of the desired gas-liquid interface inside the bulb. The new design does not allow the1/4" line to be exposed to liquid argon, thus liquid may condense inside the pipe cap, but not inside the $1 / 4^{\prime \prime}$ line.

VPT\#2 - is mounted at the equator of the inner vessel on southwest side. It is connected by $1 / 4$ " tube run up the side of the wall to port VPT2 (previously labeled as sample line port S3). The design of this bulb is depicted in D-Zero drawing \# 3740.210-MA-224761. 
VPT\#3 - is mounted just above port N11P on the southwest side. A 1/4" tube is run from the VPT out the Instrumentation port (N11P) The design of this bulb is the same as VPT\#2 and is also depicted in D-Zero drawing \# 3740.210-MA-224761.

* The previous idea was to utilize these VPT'S as gas bulbs. Research of transmitters (EN-151) uncovered the fact that no transmitter was available that could cover the range of both a gas bulb and a VPT, and still read with the desired accuracy in the VPT range. Hence the size and shape of the bulbs is no longer of great concern. 


\section{REFERENCES}

1. Mulholland, George T. Fermi National Accelerator Laboratory.

2. Peterson, Tom. Fermi National Accelerator Laboratory. 